\title{
Physical Activity Patterns and Perceptions among Type 2 Diabetic and Non-diabetic Portuguese
}

\author{
1,2Maria Dulce Leal Esteves*, ${ }^{3,4}$ Ricardo José de Ascensão Gouveia Rodrigues, ${ }^{3,4}$ Paulo \\ Gonçalves Pinheiro, ${ }^{1,2}$ Rui Miguel Marques Brás, ${ }^{5,6}$ Ana Isabel Antunes Dias Rodrigues \\ Gouveia, ${ }^{1}$ Kelly de Lemos Serrano o'Hara, ${ }^{3,4}$ Paulo Alexandre de Oliveira Duarte \\ ${ }^{1}$ Department of Sport Sciences, Rua Marquês d'Ávila e Bolama, Covilhã, Portugal. ${ }^{2}$ CIDESD (Sports \\ Sciences, Health Sciences and Human Development), Rua Marquês d'Ávila e Bolama, Covilhã, Portugal. \\ ${ }^{3}$ Department of Business and Economics, Universidade da Beira Interior, Estrada do Sineiro, Covilhã, \\ Portugal. ${ }^{4} \mathrm{NECE}$ - UBI (Research Unit in Business Sciences), Universidade da Beira Interior, Estrada do \\ Sineiro, Covilhã, Portugal. ${ }^{5}$ Faculdade de Ciências da Saúde, Universidade da Beira Interior, Covilhã, \\ Portugal. ' Instituto de Biofísica e Engenharia Biomédica, Faculdade de Ciências, Universidade de Lisboa, \\ Lisboa, Portugal.
}

Submitted 24 May 2019; Accepted in final form 15 July 2019.

\begin{abstract}
Background. The increasing prevalence and incidence of type 2 Diabetes has turned it into a worldwide epidemic. Although Physical Activity (PA) may play an essential role in the prevention and management of this disease, sedentary lifestyles continue to increase among diabetics. Besides, there is evidence to believe that diabetics and nondiabetics show different patterns of PA behavior, perceptions and information seeking. Objectives. This study aims to analyze diabetics and non-diabetics differences regarding (1) PA patterns (level, barriers, goals/expectations and preferred activities); (2) PA-related information sources and (3) evidenced perception on the need and importance of PA. Methods. Using data from 485 Portuguese subjects aged between 41 and 90 years old, differences between diabetics $(\mathrm{n}=85)$ and non-diabetics $(\mathrm{n}=400)$ were analyzed with Pearson's chi-square and independent $\mathrm{t}$-test. Results. The study concludes that $32 \%$ of diabetics that are physically inactive. Their attitude toward PA is not much different from non-diabetics. Nevertheless, the importance given to the time available and the schedules of activities as well as exercising with friends were more relevant for diabetics. Diabetics considered that the exercise is not safe for their health condition and present lower expectation towards exercise. Regarding information seeking behavior about PA, diabetics use as leading information source doctors and less online information. Conclusion. Diabetics and nondiabetics give different importance to PA. Results establish a foundation for the development of effective tailored communication strategies to promote PA among the diabetics.
\end{abstract}

\section{KEYWORDS: Diabetes, Physical Activity, Targeting}

\section{INTRODUCTION}

Diabetes has become a worldwide spread epidemic, due to the increasing prevalence and incidence of type 2 Diabetes. In 2014, 422 million people in the world had Diabetes (1): a prevalence of $8.5 \%$ among the adult population, with almost half of all deaths attributable to high blood glucose occurring before the age of 70 years (2). Diabetes is a significant cause of blindness, kidney failure, heart attacks, stroke, and lower limb amputation. However Diabetes

*. Corresponding Author:

Maria Dulce Leal Esteves, $\mathrm{PhD}$

E-mail: desteves@ubi.pt 
can be treated and its consequences avoided or delayed with diet, physical activity, medication and regular screening and treatment for complications (3). The adoption and maintenance of PA are critical for individuals with Diabetes, since exercise improves blood glucose control in type 2 Diabetes, reduces cardiovascular risk factors, contributes to weight loss and improves well-being (4). Nonetheless, sedentary lifestyle continues to increase among the diabetic population $(5,6)$. But few investigations have compared PA patterns of diabetic and non-diabetic adults. Some studies (7) focused on the comparison of the PA level of both population, pointing out a clear tendency to a lower level of PA among diabetics. Being widely confirmed this behavior presents a serious health problem as PA is crucial to increase the health status of diabetics (1) and this population shows no will to embrace an active lifestyle. The possible difference in PA patterns of diabetics and non-diabetics calls for effective strategies to promote PA among diabetics. Since the segmentation of priority populations and consistent messages as critical factors contributing to the intervention success (8) understanding how diabetics search for information seems to be crucial to design effective campaigns targeted at this population. This ideia is reinforced (4), considering that PA recommendations should be tailored to meet the specific needs of each individual. Tailored communication should be considered as an effective means to provide PA information at the individual level to adults with Diabetes, yet, there is a need to identify evidence-based factors for establishing tailored information for this population (9). Therefore, there is a need for specific, customized PA information for people with Diabetes. Given these evidences and the identified gap in the literature on the analysis of diabetics and non-diabetics behavior regarding PA information search, this investigation aims to analyze the potential to tailor PA information for diabetics on the basis of the differences from non-diabetic population regarding information seeking behavior, perceptions towards PA, and PA patterns, in order to establish the grounds to the development of effective tailored communication strategies to promote PA.

\section{MATERIALS AND METHODS}

Participants aged between 41 to 90 years old (mean=58.9; $\mathrm{SD}=11.9$ ) were included in this study, and considered diabetics $(n=85)$ if they were undergoing medical treatment with hypoglycemic agents or insulin injections (selfreported) (5). PA was assessed using the short form of the IPAQ Questionnaire (10).

\section{RESULTS}

PA Patterns. Regarding PA level assessed by IPAQ, comparing diabetics (32\% low; 25\% moderate and $44 \%$ high) and non-diabetics (29\% low; 33\% moderate and 39\% high), no statistic differences were found. Diabetics show a higher prevalence of lower and higher PA level. This result may suggest the existence of two groups among diabetics: a more active one, already engaged in PA routines and a more sedentary one $(32 \%)$ that does not meet PA recommendations.

Considering the barriers (causes of nonparticipation or drop out) to exercise, the results of diabetics and non-diabetics are presented in Table 1.

Table 1. Barriers - Causes of Non-participation or Drop out PA Programs

\begin{tabular}{|lrccc|}
\hline & Mean diabetic & Mean non-diabetic & t & p \\
Distance & 2,773 & 2,581 & 0,982 & 0,327 \\
Price & 2,853 & 3,076 & $-1,084$ & 0,253 \\
Time & 2,684 & 3,220 & $-2,721$ & $0,007 *$ \\
Timetable & 2,520 & 2,946 & $-2,294$ & $0,022 *$ \\
Transportation & 2,405 & 2,137 & 1,524 & 0,128 \\
Activities are of little interest & 2,933 & 2,798 & 0,784 & 0,433 \\
Kind / age of the people who participate & 2,568 & 2,602 & $-0,194$ & 0,846 \\
PA Monitor training/degree & 2,267 & 2,632 & $-2,045$ & $0,041^{*}$ \\
Few benefits & 2,453 & 2,314 & 0,822 & 0,412 \\
Like to do PA & 2,447 & 2,542 & $-0,531$ & 0,596 \\
Prefer doing exercise alone or with friends & 2,147 & 2,689 & $-3,161$ & $0,002 *$ \\
Participation could be dangerous to my health & 2,533 & 2,278 & 1,412 & 0,159 \\
Exercise is not adequate to their health & 2,853 & 2,437 & 2,308 & $0,021 *$ \\
Always have something else to do & 2,680 & 2,955 & $-1,495$ & 0,136 \\
I feel lazy & 2,500 & 2,529 & $-0,155$ & 0,877 \\
\hline
\end{tabular}


Table 1 shows that the frequencies for each of the barriers analyzed were lower in the case of diabetics. Except distance and lack of transportation, they are considered as a PA barrier more by diabetics (non-significant) and the perception of the few benefits of exercise and the risks it can bring to health (again, nonsignificant). Diabetics care less about the presence of friends as non-diabetics, and, a significant result, diabetics see exercise as not suitable for their health. The activity schedules and the lack of time are more significant barriers for the non-diabetic population, so when the promotion of PA is based on short, timeconsuming programs, this argument targets nondiabetics. The evaluation of goals/expectations towards exercise is presented in Table 2.

Diabetics show lower expectations about the results of participating in PA (in all items). All, except weight loss and the disease recovery show significative differences between groups. Prevention of diseases and learning healthy living habits seem to be less critical for diabetics compared with non-diabetics, nevertheless these items present higher importance among the diabetic group. It can be considered that the potentials of PA are still not sufficiently recognized by diabetics. Concerning the preferred PA (Table 3), diabetics' disfavour participation in group activities, such as aerobic classes and amateur sports. For individual PA, there are no statistically significant differences between the two groups. Diabetics prefer walking, traditional games, outdoor classes, and dance. This result is essential for the design of diabetic-specific programs.

Differences in PA-related Information Sources used by Diabetics and Non-diabetics. Considering that information about PA benefits, risks and recommendations on Diabetes disease is a crucial factor for diabetics to embrace an active lifestyle, PA information-seeking behaviour was analysed (Table 4). Doctors are the preferred source of information. Diabetic gives more importance to doctors than nodiabetic, but they rely less on Internet sites and sports professionals. Online social media is not extensively used as PA information source, neither by diabetics nor by non-diabetics. Online PA promotion campaigns, in the "real world" context, may not be the adequate delivery channel to target diabetics.

Table 2. Evaluation of the Goals/Expectations of Participation in PA

\begin{tabular}{|lcccc|}
\hline & Mean diabetic & Mean non-diabetic & $\mathbf{t}$ & $\mathbf{p}$ \\
Weight loss & 3,435 & 3,455 & $-0,135$ & 0,893 \\
Prevention of diseases & 4,012 & 4,208 & $-1,986$ & $0,048^{*}$ \\
Staying stronger and more agile & 3,167 & 3,675 & $-3,526$ & $0,000^{*}$ \\
Being with friends & 3,024 & 3,573 & $-3,835$ & $0,000^{*}$ \\
Learning healthy living habits & 3,548 & 3,938 & $-2,795$ & $0,002^{*}$ \\
Disease recovery & 3,560 & 3,760 & $-1,452$ & 0,147 \\
Recreation & 3,118 & 3,600 & $-3,540$ & $0,000^{*}$ \\
More defined muscles & 2,271 & 2,663 & $-2,676$ & $0,013^{*}$ \\
Eliminate Cellulite & 2,341 & 2,710 & $-2,153$ & $0,032^{*}$ \\
Feeling more self-confident & 2,988 & 3,373 & $-2,427$ & $0,016^{*}$ \\
Have a nicer body & 2,714 & 3,065 & $-2,166$ & $0,031^{*}$ \\
Have a younger body & 2,929 & 3,270 & $-2,208$ & $0,028^{*}$ \\
Decrease stress level & 3,214 & 3,810 & $-3,980$ & $0,000^{*}$ \\
\hline
\end{tabular}

Table 3. Preferred PA

\begin{tabular}{|lcccc|}
\hline & Mean diabetic & Mean non-diabetic & t & p \\
Walking & 3,706 & 3,818 & $-0,812$ & 0,417 \\
Gym classes machines (alone) & 1,529 & 1,890 & $-2,870$ & $0,008^{*}$ \\
Aerobics classes (in group, with music) & 1,894 & 2,355 & $-3,032$ & $0,006^{*}$ \\
Structured classes (fitness) (with guidance) & 1,706 & 2,153 & $-3,301$ & $0,003^{*}$ \\
Free classes in the gym (alone) & 1,671 & 1,915 & $-1,838$ & 0,067 \\
Outdoor classes (outdoor activities) & 2,176 & 2,774 & $-3,647$ & $0,000^{*}$ \\
Amateur sports with friends & 1,753 & 2,340 & $-4,324$ & $0,000^{*}$ \\
Structured amateur sports (with coach) & 1,753 & 2,098 & $-2,502$ & $0,013^{*}$ \\
Classes of combat modalities & 1,447 & 1,575 & $-1,110$ & 0,268 \\
Classes of mobilization / meditation & 1,667 & 1,781 & $-0,890$ & 0,374 \\
Dance classes & 2,141 & 2,445 & $-1,675$ & 0,095 \\
Extreme sports in nature & 1,518 & 1,915 & $-3,514$ & $0,004^{*}$ \\
Urban extreme sports & 1,682 & 1,915 & $-1,630$ & 0,104 \\
Traditional Games & 2,894 & 3,080 & $-1,151$ & 0,250 \\
\hline
\end{tabular}


Table 4. Information seeking and perceived Knowledge about Physical Activity

\begin{tabular}{|c|c|c|c|c|}
\hline & $\begin{array}{c}\text { Mean } \\
\text { diabetic }\end{array}$ & $\begin{array}{l}\text { Mean non- } \\
\text { diabetic }\end{array}$ & $\mathbf{t}$ & $\mathbf{p}$ \\
\hline My doctor & 3,824 & 2,977 & 4,943 & $0,000 *$ \\
\hline Friends/family & 2,882 & 2,843 & 0,261 & 0,794 \\
\hline Internet sites & 1,553 & 1,898 & $-2,831$ & $0,012 *$ \\
\hline Sports professional & 2,321 & 2,694 & $-2,144$ & $0,033 *$ \\
\hline Online social media & 1,518 & 1,673 & $-1,372$ & 0,206 \\
\hline Newspaper/magazines & 2,129 & 2,313 & $-1,210$ & 0,227 \\
\hline Know the benefits that PA brings to my life. & 3,435 & 3,712 & $-2,275$ & $0,023^{*}$ \\
\hline Would like to know more about the benefits that PA brings to my life. & 3,365 & 3,766 & $-2,822$ & $0,002 *$ \\
\hline Would like to know more about what kind of PA is right for me. & 3,388 & 3,889 & $-3,737$ & $0,000 *$ \\
\hline the information on PA I receive is correct and I trust them & 3,241 & 3,253 & $-0,090$ & 0,928 \\
\hline the information on PA I receive is not clear, and often do not understand it & 2,241 & 2,409 & $-1,260$ & 0,208 \\
\hline the information on PA I receive is not appropriate for my age/condition, so it's not useful & 2,434 & 2,404 & 0,211 & 0,833 \\
\hline
\end{tabular}

\section{DISCUSSION}

It becomes clear from the initial review conducted for this article that PA are a crucial element in the prevention and management of Diabetes $(11,12)$. However, previous research $(5$, 6) suggests that diabetics are reluctant to embrace PA programs and fight the battle with the condition to minimize the associated side effects. This evidence calls for actions to better communicate the benefits of PA among diabetics to stimulate their adherence to PA programs.

As expected the percentage of diabetics engaged in moderate or high levels of PA is slightly lower in the diabetic population (7). This low level of activity is understandable as they are conscious that they have a condition with which they must deal, and that impacts on the attendance of sports facilities. Our results point out for 32\% of diabetics that are physically inactive, with all health consequences this situation carries out (12). The first result reinforces the need for PA promotion among Portuguese diabetic population.

Regarding the barriers (causes of nonparticipation or drop out) to exercise, the most significant result is the diabetics believe that exercise is not adequate for their health. This conclusion reinforces the need for better information on PA benefits to Diabetes. Literature refers that people with Diabetes are often unwilling to participate in exercise because of specific barriers, both real and perceived ones, and patients and providers do not have enough information to take concrete actions to modify sedentary behaviour (6). Authors emphasize the need for better education and more information regarding the benefits of PA in Diabetes.

Price was also quoted as an obstacle (see Table 1), so establishing and communicating specific promotional campaigns targeting diabetics with special prices could also have a positive impact as it decreases the cost/benefits ratio. Surprisingly, despite that only five of the analyzed barriers show significant differences between the two groups, in general, the non-diabetic population report high means on the several barriers presented, with the understandable exception for the health-related obstacles. However, since the current sample does not allow for the statistical validation of the differences, further studies are needed to support the existence of divergences regarding the barriers.

Related to the previous result, when compared to non-diabetics, diabetics report lower expectations towards exercise benefits (Table 2), reinforcing the lack of awareness of PA benefits for their health condition.

The preferred activities by diabetics are the ones demanding a low or moderate level of effort, like walking, traditional games, outdoor activities and dance (Table 3). Similar results were reported by (13). A good starting point could be to base the communication and promotional strategy on the activities that are less demanding and only after the individual feels confident and secure about the benefits of the PA go further promoting more demanding sports activities. This recommendation makes good sense as the primary goals, and expectations for the PA are to prevent diseases, learning healthy living habits, disease recovery, and weight loss. By focusing on the promotion of less demanding activities among diabetics at the beginning, the risk of undesirable sideeffects diminishes, and the individual feels more self-confident on the benefits. Appearance-related issues seem to be less important.

Regarding PA information seeking, diabetics undoubtedly prefer Doctors as an information source (14). Diabetics almost do not seek PA 
information on any other source. This result underpins that any PA promotion campaign target to diabetics must involve first cares and medical personnel, in line with literature that refers health professionals as the first to recommend the adoption of new dietary and exercise behaviors among diabetics (15). Online campaigns (including the use of social media) seem to be very limited, regarding the low audience reported (see Table 4). This result is in "real world" settings, whereas literature often evaluates the effect of an online intervention considering a particular group $(16,17)$.

Non-diabetics consistently reported more to look for information about PA programs. Nevertheless, in the diabetic group, questions related to the need for information on the benefits were the ones with higher means. So, it is recommended to tailor the content of the communication to this target. For instance, by highlighting the information on the benefits, to help diabetics to overcome fears and anxieties brought about by their condition.

\section{CONCLUSION}

Based on the findings we can conclude that $32 \%$ of diabetics are physically inactive. However, they are open to engaging in PA programs as they reported to be conscious of the benefits. The risk is an important factor for them, and that is why they rely mostly on the information provided by doctors in which they trust. In general, the attitude toward PA is not much different for the diabetics and non-diabetics, except for the importance given to the time available and the schedules of activities, more critical for non-diabetics, as well as the importance of exercising with friends, more relevant for diabetics. Another notable exception is the fact that diabetics considered that the exercise is not safe for their health condition. Diabetics are also different from non-diabetics regarding information seeking behavior about PA, using as primary information source doctors and less online information comparing with nondiabetics. So, a customized communication approach is only necessary to deal with specific fears and uncertainties of diabetics, namely to help them face the worries about potential side-effects. This study presents as a main limitation the fact that the instrument used was the result of an adaptation of several questionnaires that all together are not extensively used, which makes it difficult to compare results with the literature. Besides, the application of the questionnaires has associated a series of limitations such as honesty in respondents' answers, the possibility of different understandings on the same question and lack of time to give reflected answers. These factors can condition the results obtained.

\section{APPLICABLE REMARKS}

- The present investigation comparing diabetics and non-diabetics aspires to establish a foundation for the development of effective tailored communication strategies to promote PA among the diabetics. The practical implications of the results presented can be summarized as follow:

- Providing robust and trusty evidence of the benefits and low risk associated with the exercise for diabetics is crucial, since the major barriers stated by them were the perception that exercise is not adequate to their health (18). The promotion of PA should emphasize the benefits to Diabetes and its safety.

- Lack of time and being with friends are not aspects referred to as vital by diabetics, so, PA promotion should not be grounded on these concepts.

- Light, outdoor activities seem to be preferred by diabetics.

- An important element to be considered is to decide who should provide counseling and evidence. In line with previous research (19), current results show that doctors are the best channel to convey information to diabetics. Results from non-diabetics are similar.

- Online PA promotion campaigns must be conveyed with personal information to be a specific target to that individual. Otherwise they are inefficient.

- Primary care practitioners can play a key role in promoting PAamong diabetics, both delivering adequate information and encouraging diabetics to become more active (20). Consider that PA promotion based on technology must be viewed as a potential solution of delivering tailored information. 


\section{REFERENCES}

1. World Health Organization. Global Report on Diabetes: WHO; 2016 [cited 2018 May 3]. Available from: http://www.who.int/iris/handle/10665/204871.

2. Mathers CD, Loncar D. Projections of global mortality and burden of disease from 2002 to 2030. PLoS Med. 2006;3(11):e442. doi: 10.1371/journal.pmed.0030442 pmid: 17132052

3. Asif $\mathrm{M}$. The prevention and control the type-2 diabetes by changing lifestyle and dietary pattern. J Educ Health Promot. 2014;3:1. doi: 10.4103/2277-9531.127541 pmid: 24741641

4. Colberg SR, Sigal RJ, Yardley JE, Riddell MC, Dunstan DW, Dempsey PC, et al. Physical Activity/Exercise and Diabetes: A Position Statement of the American Diabetes Association. Diabetes Care. 2016;39(11):2065-2079. doi: 10.2337/dc16-1728 pmid: 27926890

5. Iwasa T, Amiya E, Ando J, Watanabe M, Murasawa T, Komuro I. Different Contributions of Physical Activity on Arterial Stiffness between Diabetics and Non-Diabetics. PLoS One. 2016;11(8):e0160632. doi: 10.1371/journal.pone.0160632 pmid: 27508936

6. Jenkins DW, Jenks A. Exercise and Diabetes: A Narrative Review. J Foot Ankle Surg. 2017;56(5):968-974. doi: 10.1053/j.jfas.2017.06.019 pmid: 28842107

7. Das I, Chardhary S. Physical Activity of Diabetics and Non-Diabetics. In: Agarwala S, Ira D, Kumar K, Kumar S, editors. Health Psychology: Allied Publishers; 2016. p. 71-81.

8. Gallivan J, Lising M, Ammary NJ, Greenberg R. The National Diabetes Education Program's "Control Your Diabetes. For Life." Campaign: Design, Implementation, and Lessons Learned. Soc Market Q. 2016;13(4):65-82. doi: 10.1080/15245000701678453

9. Enwald HP, Niemela RM, Keinanen-Kiukaanniemi S, Leppaluoto J, Jamsa T, Herzig KH, et al. Human information behaviour and physiological measurements as a basis to tailor health information. An explorative study in a physical activity intervention among prediabetic individuals in Northern Finland. Health Info Libr J. 2012;29(2):131-140. doi: 10.1111/j.14711842.2011.00968.x pmid: 22630361

10. Hagstromer M, Oja P, Sjostrom M. The International Physical Activity Questionnaire (IPAQ): a study of concurrent and construct validity. Public Health Nutr. 2006;9(6):755-762. pmid: 16925881

11. Colberg SR, Sigal RJ, Fernhall B, Regensteiner JG, Blissmer BJ, Rubin RR, et al. Exercise and type 2 diabetes: the American College of Sports Medicine and the American Diabetes Association: joint position statement. Diabetes Care. 2010;33(12):e147-167. doi: 10.2337/dc10-9990 pmid: 21115758

12. Sabag A, Way KL, Keating SE, Sultana RN, O'Connor HT, Baker MK, et al. Exercise and ectopic fat in type 2 diabetes: A systematic review and meta-analysis. Diabetes Metab. 2017;43(3):195-210. doi: 10.1016/j.diabet.2016.12.006 pmid: 28162956

13. Booth ML, Bauman A, Owen N, Gore CJ. Physical activity preferences, preferred sources of assistance, and perceived barriers to increased activity among physically inactive Australians. Prev Med. 1997;26(1):131-137. doi: 10.1006/pmed.1996.9982 pmid: 9010908

14. Jamal A, Khan SA, AlHumud A, Al-Duhyyim A, Alrashed M, Bin Shabr F, et al. Association of Online Health Information-Seeking Behavior and Self-Care Activities Among Type 2 Diabetic Patients in Saudi Arabia. $J$ Med Internet Res. 2015;17(8):e196. doi: 10.2196/jmir.4312 pmid: 26268425

15. Plotnikoff RC, Johnson ST, Karunamuni N, Boule NG. Physical activity related information sources predict physical activity behaviors in adults with type 2 diabetes. J Health Commun. 2010;15(8):846-858. doi: 10.1080/10810730.2010.522224 pmid: 21170787

16. Connelly J, Kirk A, Masthoff J, MacRury S. The use of technology to promote physical activity in Type 2 diabetes management: a systematic review. Diabet Med. 2013;30(12):14201432. doi: 10.1111/dme. 12289 pmid: 23870009

17. Harris JK, Mueller NL, Snider D, Haire-Joshu D. Local health department use of twitter to disseminate diabetes information, United States. Prev Chronic Dis. 2013;10:E70. doi: 10.5888/pcd10.120215 pmid: 23639765 
18. Korkiakangas EE, Alahuhta MA, Laitinen JH. Barriers to regular exercise among adults at high risk or diagnosed with type 2 diabetes: a systematic review. Health Promot Int. 2009;24(4):416-427. doi: 10.1093/heapro/dap031 pmid: 19793763

19. Sanchez A, Bully P, Martinez C, Grandes G. Effectiveness of physical activity promotion interventions in primary care: A review of reviews. Prev Med. 2015;76 Suppl:S56-67. doi: 10.1016/j.ypmed.2014.09.012 pmid: 25263343

20. Rehman H, Kamal AK, Sayani S, Morris PB, Merchant AT, Virani SS. Using Mobile Health (mHealth) Technology in the Management of Diabetes Mellitus, Physical Inactivity, and Smoking. Curr Atheroscler Rep. 2017;19(4):16. doi: 10.1007/s11883-017-0650-5 pmid: 28243807 